\title{
On-chip Fourier-transform spectrometers and machine learning: a new route to smart photonic
} sensors

\author{
ALAINE HERRERO-BERMELLO,,$^{1, *}$ JIANGFENG $L I,{ }^{2}$ MOHAMMAD \\ KHAZAEI, ${ }^{2}$ YURI GRINBERG, ${ }^{3}$ AITOR V. VELASCO, ${ }^{1}$ MARTIN VACHON, ${ }^{3}$ \\ PAVEL CHEBEN, ${ }^{3}$ LINA STANKOVIC, ${ }^{2}$ VLADIMIR STANKOVIC, ${ }^{2}$ DAN-XIA \\ XU, ${ }^{3}$ JENS H. SCHMID, ${ }^{3}$ AND CARLOS ALONSO-RAMOS ${ }^{4}$
}

${ }^{1}$ Instituto de Óptica, Consejo Superior de Investigaciones Científicas, Madrid 28006, España.

${ }^{2}$ Department of Electronic and Electrical Engineering, University of Strathclyde, Glasgow G1 1XW, U.K.

${ }^{3}$ National Research Council of Canada, Ottawa, Ontario, K1A OR6, Canada.

${ }^{4}$ Centre de Nanosciences et de Nanotechnologies, CNRS, Univ. Paris-Sud, Université Paris-Saclay, 91405 Orsay cedex, France.

*Corresponding author: alaine.herrero@csic.es

Received XX Month XXXX; revised XX Month, XXXX; accepted XX Month XXXX; posted XX Month XXXX (Doc. ID XXXXX); published XX Month XXXX

Miniaturized silicon photonics spectrometers capable of detecting specific absorption features have great potential for mass market applications in medicine, environmental monitoring, and hazard detection. However, state-of-the-art silicon spectrometers are limited by fabrication imperfections and environmental conditions, especially temperature variations, since uncontrolled temperature drifts of only $0.1^{\circ} \mathrm{C}$ distort the retrieved spectrum precluding the detection and classification of the absorption features. Here, we present a new strategy that exploits the robustness of machine learning algorithms to signal imperfections, enabling recognition of specific absorption features in a wide range of environmental conditions. We combine onchip spatial heterodyne Fourier-transform spectrometers and supervised learning to classify different input spectra in the presence of fabrication errors, without temperature stabilization or monitoring. We experimentally show differentiation of four different input spectra under an uncontrolled $10{ }^{\circ} \mathrm{C}$ range of temperatures, about 100x increase in operational range, with a success rate up to $82.5 \%$ using state-of-the-art support vector machines and artificial neural networks. (C) 2019 Optical Society of America

http://dx.doi.org/10.1364/OL.99.099999

Miniaturized silicon photonics spectrometers have a great potential for mass market applications, ranging from medicine, biological and environmental sciences to astrophysics and telecommunication [1]. Most common approaches, based on dispersive devices such as arrayed waveguide gratings (AWG) [2], waveguide echelle gratings [3] or cascaded micro-ring resonators [4], present some important disadvantages that limit their performance in terms of sensitivity to fabrication imperfections and environmental conditions, signal-to-noise ratio (SNR) and optical throughput. Most of these limitations are overcome with spatial heterodyne Fourier-transform (SHFT) spectrometers [511], which provide the high SNR and optical throughput of conventional Fourier-transform (FT) spectrometers [12], while obviating the need of moving elements or heaters [13]. SHFT spectrometers rely on an array of Mach-Zehnder interferometers (MZI), each of them with a different optical path difference (OPD) to create a spatial interferogram from which the input spectrum is retrieved [5].

This approach enables passive calibration techniques that correct the effects of fabrication imperfections [5,14]. However, even if fabrication errors are corrected, additional thermallyinduced phase errors can arise due to the high thermal dependence of the Si waveguides, $d n_{\text {eff }} / d T$ (which is equal to $1.8 \cdot 10^{-4} \mathrm{~K}^{-1}$ and $1.2 \cdot 10^{-4} \mathrm{~K}^{-1}$, for TE and TM polarization, respectively, at a wavelength of $1.55 \mu \mathrm{m}$ ) [15]. This thermal dependence imposes a rigorous temperature control. For a $42 \mathrm{pm}$ resolution spectrometer [5], as the one presented in Fig. 1, temperature variations as small as $0.1^{\circ} \mathrm{C}$ produce alterations in the OPD of each interferometer that result in significant degradation of the retrieved input spectrum, including central wavelength shifts, sidelobe level increments and presence of artifacts (see Fig. 1 (c)). A resolution of $42 \mathrm{pm}\left(0.16 \mathrm{~cm}^{-1}\right)$ approaches the requirements for gas-phase detection [16]. However, the strong temperature 
dependence limits the applicability of conventional on-chip SHFT spectrometers outside of the lab. In [15], spectral retrieval methods based on a temperature-sensitive calibration are presented to mitigate this problem. However, these methods rely on extensive and accurate calibration and auxiliary temperature measurements in order to accurately retrieve the complete spectrum, which can be burdensome in applications such as gas identification, which only require robust classification among a fixed set of inputs in a wide range of operation conditions. Concurrently, machine learning (ML) has already been successfully applied to analyze complex data creating predictive models for pattern classification in a plethora of applications, including facial expression recognition [17], cancer prognosis and prediction [18], seismic wave discrimination [19], genetic and genomic analysis [20] or automated traffic classification [21]. All these applications benefit from the unique capability of ML algorithms to detect and classify specific patterns, even under changing or unknown environmental conditions or noise.

In this work, we present a new spectral recognition approach that leverages ML for SHFT spectrometers to recognize specific absorption features in the presence of fabrication imperfections, regardless of temperature. We train our algorithm to recognize the signature of specific absorption patterns directly from the analysis of the output interferogram of a conventional SHFT spectrometer, circumventing the need to calculate the input spectrum. This training is performed for a specific chip, designed for conventional operation, with given fabrication imperfections (e.g. deviations in waveguide dimensions and splitting ratio) and a wide range of temperatures, rendering our system robust against these parameters. As a proof of concept, we experimentally demonstrate recognition of four different input spectra using the on-chip SHFT spectrometer presented in [5] (Fig. 1) but with unknown temperature within a $10{ }^{\circ} \mathrm{C}$ range (that is, a 100x increase compared to the original operational temperature range of the device).

In SHFT spectrometers the output interferogram, $\mathbf{I}\left(x_{i}\right)$, sampled at OPDs $x_{i}$, contains spatially distributed interferometric information of the spectral density of the input signal B. Output interferogram and input spectrum are related by [5]:

$$
\mathbf{I}\left(x_{i}\right)=\mathbf{B} \times \mathbf{T},
$$

where $\mathbf{T}$ is a transformation matrix containing the measured transmittance of each MZI. The input power spectrum $\mathbf{B}$ is retrieved by multiplying the spatial interferogram $\mathbf{I}\left(x_{i}\right)$ by $\mathbf{T}^{+}$, which is a pseudoinverse of the transformation matrix $\mathbf{T}$. While this approach corrects amplitude and phase errors produced by fabrication imperfections, the issue is that the matrix $\mathbf{T}$ strongly depends on the temperature. Thus, input spectrum retrieval requires accurate knowledge of the temperature or precise temperature stabilization to apply the correct $\mathbf{T}^{+}$matrix. ML algorithms have already been used to optimize the calculation of the pseudoinverse of the matrix $\mathbf{T}$ [14]. However, these approaches do not address the temperature dependence issue.

To overcome this challenge, we propose the application of ML techniques to build a mathematical model based on the analysis of the measured output interferogram $\mathbf{I}\left(x_{i}\right)$, without retrieving the input spectrum B. We apply a supervised learning scheme that relies on a set of labeled samples (training data) to find the internal structure of the information, i.e., a pattern, and then predict the label or class of other samples (test data). In our case, the labels correspond to different known input spectra. The labeled data are the values of the output interferogram, measured when these known input spectra are coupled into the spectrometer with different unknown temperatures within a $10^{\circ} \mathrm{C}$ range. The task is to train the ML algorithm on one subset of labeled data and be able to classify on multiple "unseen" datasets obtained in different experiments, with different temperatures. We resorted to supervised transfer learning based on support vector machine (SVM) and artificial neural network (ANN). These methods require low training and testing complexity even for very high dimensional features and have been proven to perform well when training data are scarce.

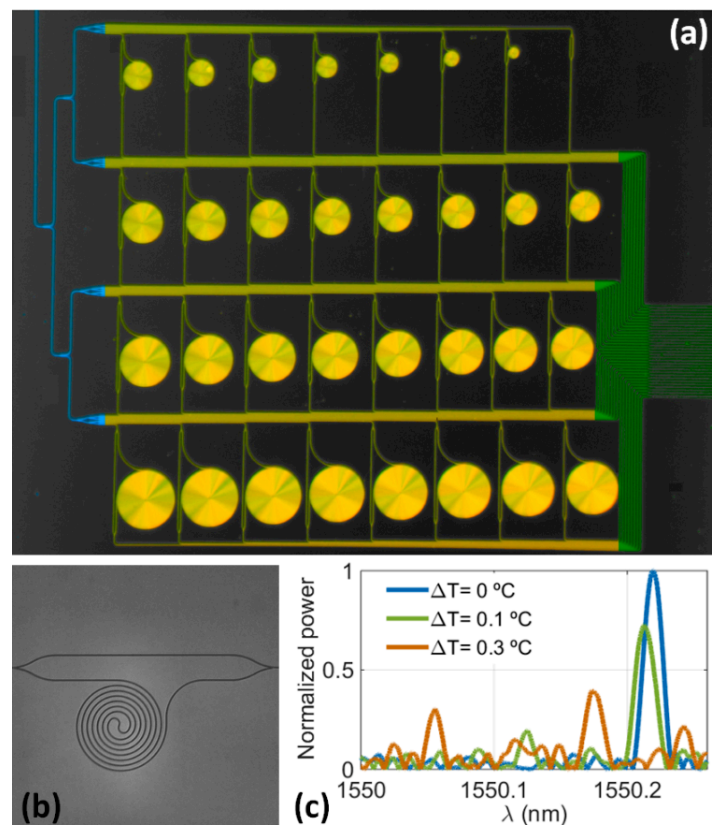

Fig. 1. (a) SEM image of the spatial heterodyne Fourier-transform spectrometer under analysis. (b) Enlarged and detailed image of one Mach-Zehnder interferometer. (c) Effects of temperature variations in the spectral retrieval of a monochromatic input signal.

To experimentally demonstrate the proposed approach, we used a SHFT micro-spectrometer fabricated on silicon-oninsulator (SOI) wafers with 260-nm-thick silicon and $2 \mu \mathrm{m}$ buried oxide (BOX) [5]. The device (Fig. 1) comprises an array of 31 silicon waveguide MZIs in which the OPD is implemented through tightly coiled micro-photonic spirals of linearly increasing length up to $1.13 \mathrm{~cm}$, with a maximum diameter of only $270 \mu \mathrm{m}$ and minimum bend radius of $5 \mu \mathrm{m}$. Si-wire waveguides were selected to be 450 -nm-wide $\mathrm{x} 260$-nm-thick to ensure single mode operation and minimize scattering losses. Efficient sub-wavelength grating edge couplers [22] were integrated on the chip for fiberchip coupling, while at the same time reducing the Fabry-Perot cavity effect by minimizing the reflectivity at the facets. Waveguides were defined in a single patterning step by electron beam lithography using hydrogen silsesquioxane (HSQ) resist. Inductively coupled plasma reactive ion etching was used to transfer the resist pattern into the silicon layer. The resulting propagation losses in the waveguides were measured to be -4 $\mathrm{dB} / \mathrm{cm}$ and the bending losses in the spiral sections $-1.7 \mathrm{~dB} / \mathrm{cm}$. We defined a target classification problem with four classes of 
output interferogram signals: the first three classes consist of single absorption lines at selected wavelengths within the free spectral range of the spectrometer (1549.8, 1550.0 and 1550.2 $\mathrm{nm}$ ), while the fourth class corresponds to a reference signal in which no absorption line is present (see Fig. 2). For the generation of the input signals, a programmable spectral processor with a pass band of $750 \mathrm{pm}$ and a tunable fiber Bragg grating (FBG) were used. The interferogram was measured for the TM-polarization state, selected through an external polarization controller, and output light from the MZIs was collected by a $5 \mathrm{x}$ microscope objective and captured in a single shot with a high-sensitivity InGaAs camera. (a)
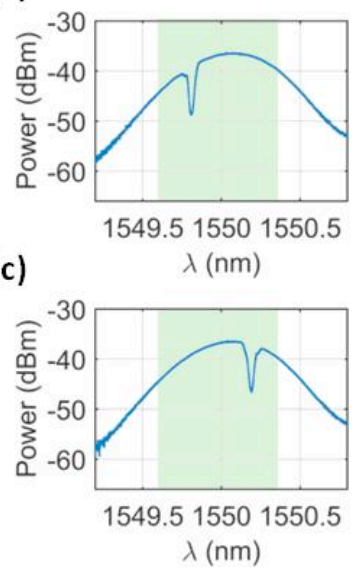

(b)
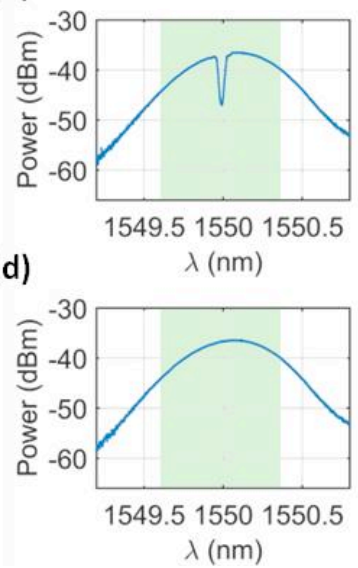

Fig. 2. Optical spectrum of the three classes of output interferogram signals with single absorption lines at $1549.8 \mathrm{~nm}(\mathrm{a}), 1550.0 \mathrm{~nm}(\mathrm{~b})$ and $1550.2 \mathrm{~nm}$ (c) and of the reference signal without absorption line (d). The green shaded band corresponds to the $0.75 \mathrm{~nm}$ wide free spectral range of the spectrometer.

In the experimental step only 28 interferometers (from MZI \#2 to MZI \#29) could be measured simultaneously, due to the limited size of the camera's detector area (see Fig. 3(a)). The values of the output interferogram were calculated by fitting a Gaussian to several adjacent pixel values around the position of the actual peak. We measured several interferograms for each input spectrum, while varying the temperature of the chip in a $10{ }^{\circ} \mathrm{C}$ range (from $20{ }^{\circ} \mathrm{C}$ to $30{ }^{\circ} \mathrm{C}$ ) using a Peltier stage. Each measurement resulted in one data point that takes the form of a vector of 28 optical power values, $\mathbf{I}\left(x_{i}\right)$ (Fig. 3(b)). We collected a total of 4655 data points for each of the four input spectra (classes). Half the data points were used for training and half for testing of the algorithm.

For the SVM, we opted for the Gaussian kernel since it is the most popular general purpose function to transform the input data into a well-defined feature space. For the ANN, we trained the network with Broyden, Fletcher, Goldfarb, and Shanno (BFGS) quasi-Newton backpropagation and Levenberg-Marquardt backpropagation. The number of hidden layers we experimented ranged from 10 to 50 . These were further refined through feature selection, i.e., determining which of the 28 optical values carried more weight in helping infer the correct class.
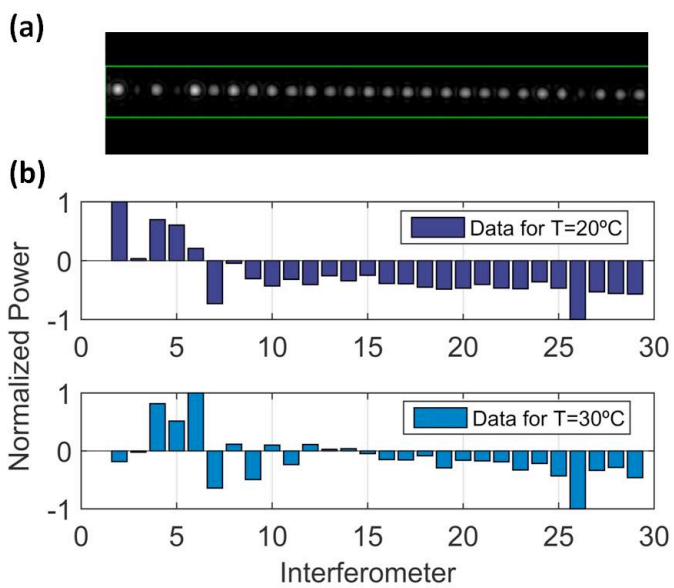

Fig. 3. (a) High-sensitivity InGaAs camera image of the output of the 28 MZIs at a temperature of $20^{\circ} \mathrm{C}$, (b) normalized output interferogram of the 28 MZIs at a temperature of $20^{\circ} \mathrm{C}$ (upper panel) and $30^{\circ} \mathrm{C}$ (lower panel), for a $1551.5 \mathrm{~nm}$ laser input signal.

In order to demonstrate the classification challenge, we plot, from the testing set, the mean of the 28 optical power values $\mathbf{I}\left(x_{i}\right)$ for each experiment (Fig. 4). Deviations in mean of measurements are apparent across samples/experiments, while the first three classes (single absorption lines) present very similar-valued datapoints. Class 4 is therefore expected to be most accurately classified, as it is less likely to be confused with other 3 classes which have similar power values. On the other hand, Classes 1, 2 and 3 are more difficult to separate.

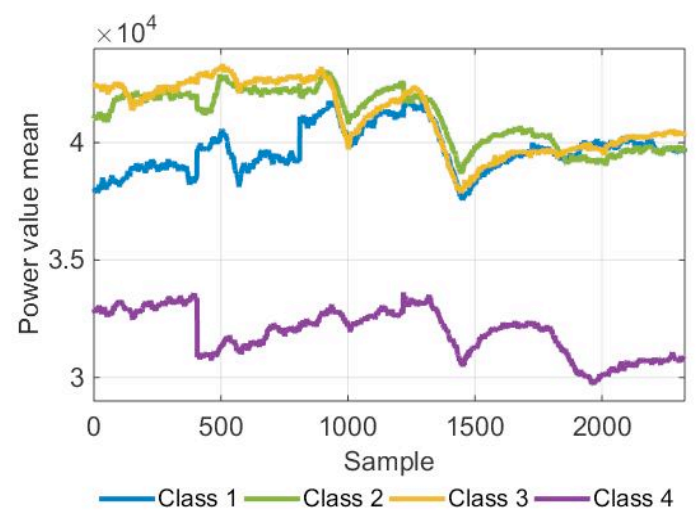

Fig. 4. Magnitude of average power values for each of the 4 classes, $1549.8 \mathrm{~nm}, 1550.0 \mathrm{~nm}, 1550.2 \mathrm{~nm}$ and no peak, respectively, measured in the $20-30{ }^{\circ} \mathrm{C}$ temperature range.

Classification accuracy in ML is typically represented by the F1 score which is a weighted average of precision and recall metrics, taking into account true positives (TP), false positives (FP) and false negatives $(\mathrm{FN})$. Precision is given by $\mathrm{TP} /(\mathrm{TP}+\mathrm{FP})$, i.e., the ratio of correctly predicted positive observations to the total predicted positive observations. Recall is the ratio of correctly predicted positive observations to all the observations in the actual class and is given by $\mathrm{TP} /(\mathrm{TP}+\mathrm{FN})$. $\mathrm{F} 1$ score is then calculated for each class by $2 *$ (Recall*Precision)/(Recall+Precision), and then the results are averaged over all four classes. In the first instance, classification was performed using the full vector of 28 spectrometer outputs. 
This resulted in an F1 score accuracy of $77.6 \%$ for SVM and $77.8 \%$ for ANN, when tested on $2327 \times 4$ data points. The best results for ANN were obtained when training was performed with Levenberg-Marquardt backpropagation with 35 hidden layers.

Next, feature selection showed that filtering out the following MZI vector of measurements, resulted in the best performance: for SVM $\mathbf{I}\left(x_{3}\right), \mathbf{I}\left(x_{6}\right), \mathbf{I}\left(x_{9}\right), \mathbf{I}\left(x_{11}\right), \mathbf{I}\left(x_{16}\right), \mathbf{I}\left(x_{21}\right), \mathbf{I}\left(x_{24}\right), \mathbf{I}\left(x_{25}\right)$ were filtered out and for ANN I $\left(x_{23}\right)$ was filtered out. Following feature selection and classification, we analyze the confusion matrix that shows which predictions were correct (TP) along the diagonal (i.e., actual vs predicted class 1 , actual vs. predicted class 2 etc.), but also indicates which other classes were incorrectly classified or confused with each other. The improved results with feature selection are shown as confusion matrices in Tables 1 and 2, evaluated on the test set of $2327 \times 4$ data points and corresponding to accuracy of $81.3 \%$ and $82.5 \%$ for ANN and SVM, respectively. As expected from the observation in Fig. 4, both tables show that there is a not-insignificant misclassification of class 3 as class 2 (Table 1) and class 2 as class 1 (Table 2), i.e., strong confusion in correctly identifying some samples in classes 1, 2 and 3. Similarly, class 4 is hardly misclassified as other classes (especially Table 2 with zero misclassification). However, the diagonal line in both tables still indicates that both algorithms correctly classify the vast majority of all samples, showing robustness of the ML algorithms in correctly retrieving the spectra. Further improvements in accuracy and misclassification may be achieved in the future by exploring more advanced feature selection and classification methods, and by including additional measurements under varying environmental conditions.

Table 1. ANN classification: best results confusion matrix.

\begin{tabular}{|c|c|c|c|c|}
\hline $\begin{array}{l}\text { Predicted } \\
\text { Actual }\end{array}$ & class 1 & class 2 & class 3 & class 4 \\
\hline class 1 & 1782 & 383 & 161 & 1 \\
\hline class 2 & 90 & 1869 & 318 & 49 \\
\hline class 3 & 0 & 676 & 1647 & 1 \\
\hline class 4 & 1 & 39 & 62 & 2222 \\
\hline
\end{tabular}

Table 2. SVM classification: best results confusion matrix.

\begin{tabular}{|c|c|c|c|c|}
\hline $\begin{array}{c}\text { Predicted } \\
\text { Actual }\end{array}$ & class 1 & class 2 & class 3 & class 4 \\
\hline class 1 & 1523 & 343 & 461 & 0 \\
\hline class 2 & 361 & 1622 & 343 & 0 \\
\hline class 3 & 133 & 5 & 2186 & 0 \\
\hline class 4 & 0 & 0 & 0 & 2324 \\
\hline
\end{tabular}

In conclusion, this proof of concept has demonstrated the excellent potential of ML techniques for interferogram classification in on-chip FT spectrometers. While state-of-the-art spectral retrieval techniques for on-chip SHFT spectrometers require temperature control within $0.1^{\circ} \mathrm{C}$ to retrieve input spectrum enabling feature classification, ML algorithms are capable of detecting the underlying characteristic signatures of absorption features at the input of the spectrometer, regardless of interferogram changes caused by environmental effects, and consequently, circumvent the stringent requirements on their thermal stabilization system. In particular, the accuracy of the popular supervised SVM and ANN algorithms have been studied in a SHFT spectrometer fabricated on silicon-on-insulator. The device under analysis comprises an array of 31 MZIs with microphotonic spirals of linearly increasing length in a footprint of only $12 \mathrm{~mm}^{2}$. The ANN and SVM methods successfully classified four different classes under varying temperature conditions, obtaining accuracy of $78 \%$ without feature selection. This value improves up to $82.5 \%$ with feature selection. The results obtained by the synergic combination of microspectrometers and ML pave the way for future optimizations of classification accuracy yielding to a new generation of smart miniaturized silicon photonic sensors.

Funding. Spanish Ministry of Science, Innovation and Universities (RTI2018-097957-B-C33, IJCI-2016-30484); Community of Madrid - FEDER funds (S2018/NMT-4326); The Horizon 2020 research and innovation program (Marie Sklodowska-Curie RISE Action 734331); Project MIRSPEC (ANR-17-CE09-0041).

\section{References}

1. M. Ferrari, and V. Quaresima, Neuroimage 63, 921 (2012).

2. B. I. Akca, and C. R. Doerr, IEEE Photon. Technol. Lett. 31, 90 (2018).

3. K. Ma, K. Chen, N. Zhu, L. Liu, and S. He, IEEE Photon. J. 11, 4900107 (2018).

4. J. Huang, J. Yang, H. Zhang, J. Zhang, W. Wu, and S. Chang, IEEE Photon. Technol. Lett. 28, 2677 (2016).

5. A. V. Velasco, P. Cheben, P. J. Bock, A. Delâge, J. H. Schmid, J. Lapointe, S. Janz, M. L. Calvo, D.-X. Xu, M. Florjanczyk, and Martin Vachon, Opt. Lett. 38, 706 (2013).

6. H. Podemore, A. Scott, P. Cheben, A. V. Velasco, J. H. Schmid, M. Vachon, and R. Lee, Opt. Lett. 42, 1440 (2017).

7. K. Okamoto, H. Aoyagi, and K. Takada, Opt. Lett. 35(12), 2103 (2010).

8. E. L. Coarer, S. Blaize, P. Benech, I. Stefanon, A. Morand, G. Lérondel, G. Leblond, P. Kern, J. M. Fedeli, and P. Royer, Nat. Photon. 1, 473 (2007).

9. X. Nie, E. Ryckeboer, G. Roelkens, and R. Baets, Opt. Express 25, A409 (2017).

10. R. A. Soref, F. D. Leonardis, and V. M. N. Passaro, J. Light. Technol. 37, 3192 (2019).

11. B. I. Akca, Opt. Express 25, 1487 (2017).

12. P. R. Griffiths, J. Appl. Spectrosc. 71, 329 (2017).

13. M. C. M. M. Souza, A. Grieco, N. C. Frateschi, and Y. Fainman, Nat. Commun. 9, 665 (2018).

14. D. M. Kita, B. Miranda, D. Favela, D. Bono, J. Michon, H. Lin, T. Gu, and J. Hu, Nat. Commun. 9, 4405 (2018).

15. A. Herrero-Bermello, A. V. Velasco, H. Podmore, P. Cheben, J. H. Schmid, S. Janz, M. L. Calvo, D.-X. Xu, A. Scott, and P. Corredera, Opt. Lett. 42, 2239 (2017).

16. S. W. Sharpe, T. J. Johnson, R. L. Sams, P. M. Chu, G. C. Rhoderick, and P. A. Johnson, Appl. Spectrosc. 58, 1452 (2005).

17. M. S. Bartlet, G. Littlewort, M. Frank, C. Lainscsek, I. Fasel, and J. Movellan, in Proceedings of IEEE Computer Society Conference on Computer Vision and Pattern Recognition (IEEE, 2005).

18. P. Lakhani, A. B. Prater, R. K. Hutson, K. P. Andriole, K. J. Dreyer, J. Morey, L. M. Prevedello, T. J. Clark, J. R. Geis, J. N. Itri, and C. M. Hawkins, J. Am. Coll. Radiol. 15, 350 (2018).

19. Z. Li, M. A. Meier, E. Hauksson, Z. Zhan, and J. Andrews, Geophys. Res. Lett. 45, 4773 (2018).

20. M. W. Libbrecht, and W. S. Noble, Nature Rev. Genet. 16, 321 (2015).

21. S. Zander, T. Nguyen, and G. Armitage, in Proceedings of IEEE Conference on Local Computer Networks (IEEE, 2005).

22. P. Cheben, P. J. Bock, J. H. Schmid, J. Lapointe, S. Janz, D.-X. Xu, A. Densmore, A. Delâge, B. Lamontagne, and T. J. Hall, Opt. Lett. 35, 2526 (2010). 


\section{REFERENCES}

1. M. Ferrari, and V. Quaresima, "A brief review on the history of human functional near-infrared spectroscopy (fNIRS) development and fields of application," Neuroimage 63, 921-935 (2012).

2. B. I. Akca, and C. R. Doerr, "Interleaved silicon nitride AWG spectrometers," IEEE Photon. Technol. Lett. 31, 90-93 (2018).

3. S K. Ma, K. Chen, N. Zhu, L. Liu, and S. He, "High-resolution compact onchip spectrometer based on an echelle grating with densely packed waveguide array," IEEE Photon. J. 11, 4900107 (2018).

4. J. Huang, J. Yang, H. Zhang, J. Zhang, W. Wu, and S. Chang, "Analysis of tunable flat-top bandpass filters based on graphene," IEEE Photon. Technol. Lett. 28, 2677-2680 (2016).

5. A. V. Velasco, P. Cheben, P. J. Bock, A. Delâge, J. H. Schmid, J. Lapointe, S. Janz, M. L. Calvo, D.-X. Xu, M. Florjanczyk, and Martin Vachon, "Highresolution Fourier-transform spectrometer chip with microphotonic silicon spiral waveguides," Opt. Lett. 38, 706-708 (2013).

6. H. Podemore, A. Scott, P. Cheben, A. V. Velasco, J. H. Schmid, M. Vachon, and R. Lee, "Demonstration of a compressive-sensing Fourier-transform on-chip spectrometer," Opt. Lett. 42, 1440-1443 (2017).

7. K. Okamoto, H. Aoyagi, and K. Takada, "Fabrication of Fourier-transform, integrated-optic spatial heterodyne spectrometer on silica-based planar waveguide," Opt. Lett. 35, 2103-2105 (2010).

8. E. L. Coarer, S. Blaize, P. Benech, I. Stefanon, A. Morand, G. Lérondel, G. Leblond, P. Kern, J. M. Fedeli, and P. Royer, "Wavelength-scale stationarywave integrated Fourier-transform spectrometry," Nat. Photon. 1, 473478 (2007).

9. X. Nie, E. Ryckeboer, G. Roelkens, and R. Baets, "CMOS-compatible broadband co-propagative stationary Fourier transform spectrometer integrated on a silicon nitride photonics platform," Opt. Express 25, A409A418 (2017).

10. R. A. Soref, F. D. Leonardis, and V. M. N. Passaro, "Scanning spectrometer-on-a-chip using thermo-optical spike-filters or Verniercomb filters," J. Light. Technol. 37, 3192-3200 (2019).

11. B. I. Akca, "Design of a compact and ultrahigh-resolution Fouriertransform spectrometer," Opt. Express 25, 1487-1494 (2017).

12. P. R. Griffiths, "The early days of commercial FT-IR spectrometry: a personal perspective," J. Appl. Spectrosc. 71, 329-340 (2017).

13. M. C. M. M. Souza, A. Grieco, N. C. Frateschi, and Y. Fainman, "Fourier transform spectrometer on silicon with thermo-optic non-linearity and dispersion correction," Nat. Commun. 9, 665 (2018).

14. D. M. Kita, B. Miranda, D. Favela, D. Bono, J. Michon, H. Lin, T. Gu, and J. $\mathrm{Hu}$, "High-performance and scalable on-chip digital Fourier transform spectroscopy," Nat. Commun. 9, 4405 (2018).

15. A. Herrero-Bermello, A. V. Velasco, H. Podmore, P. Cheben, J. H. Schmid, S. Janz, M. L. Calvo, D.-X. Xu, A. Scott, and P. Corredera, "Temperature dependence mitigation in stationary Fourier-transform on-chip spectrometers," Opt. Lett. 42, 2239-2242 (2017).

16. S. W. Sharpe, T. J. Johnson, R. L. Sams, P. M. Chu, G. C. Rhoderick, and P. A. Johnson, "Gas-phase databases for quantitative infrared spectroscopy," Appl. Spectrosc. 58, 1452-1461 (2005).

17. M. S. Bartlet, G. Littlewort, M. Frank, C. Lainscsek, I. Fasel, and J. Movellan, "Recognizing facial expression: machine learning and application to spontaneous behavior," in Proceedings of IEEE Computer Society Conference on Computer Vision and Pattern Recognition (IEEE, 2005).

18. P. Lakhani, A. B. Prater, R. K. Hutson, K. P. Andriole, K. J. Dreyer, J. Morey, L. M. Prevedello, T. J. Clark, J. R. Geis, J. N. Itri, and C. M. Hawkins, "Machine learning in radiology: applications beyond image interpretation," J. Am. Coll. Radiol. 15, 350-359 (2018).

19. Z. Li, M. A. Meier, E. Hauksson, Z. Zhan, and J. Andrews, "Machine learning seismic wave discrimination: application to earthquake early warning," Geophys. Res. Lett. 45, 4773-4779 (2018).

20. M. W. Libbrecht, and W. S. Noble, "Machine learning applications in genetics and genomics," Nature Rev. Genet. 16, 321-332 (2015).
21. S. Zander, T. Nguyen, and G. Armitage, "Automated traffic classification and application identification using machine learning," in Proceedings of IEEE Conference on Local Computer Networks (IEEE, 2005).

22. P. Cheben, P. J. Bock, J. H. Schmid, J. Lapointe, S. Janz, D.-X. Xu, A. Densmore, A. Delâge, B. Lamontagne, and T. J. Hall, "Refractive index engineering with subwavelength gratings for efficient microphotonic couplers and planar waveguide multiplexers," Opt. Lett. 35, 2526-2528 (2010). 